\title{
EVALUATION OF CLINICALLY IMPORTANT GRAM-NEGATIVE ISOLATES (ESCHERICHIA COLI, KLEBSIELLA PNEUMONIAE, PSEUDOMONAS AERUGINOSA) AND THEIR SUSCEPTIBILITY PATTERN IN TERTIARY CARE HOSPITAL: 3 YR PROSPECTIVE STUDY
}

\author{
Ram Murgan Navaneethkrishnan ${ }^{1}$, Sugumari Chandrasegaran ${ }^{2}$
}

${ }^{1}$ Senior Assistant Professor, Department of Microbiology, Institute of Microbiology, Madurai Medical College, Madurai. 2Senior Assistant Professor, Department of Microbiology, Institute of Microbiology, Madurai Medical College, Madurai.

ABSTRACT
AIM
1. To analyse the clinically important Gram-negative isolates (Escherichia coli, Klebsiella pneumoniae and Pseudomonas
aeruginosa) and their susceptibility pattern in a tertiary care hospital; 2. To study the epidemiology of the isolates and sensitivity
pattern of the Gram negative bacteria in a tertiary care hospital, Madurai, South Tamilnadu.

\section{METHODS}

Samples collected from different wards were subjected for culture, isolation and identification of Gram negative bacterial isolates and antibacterial susceptibility testing by Vitek System and Kirby Bauer method. This study was carried out in Apollo Speciality Hospital, Lab Services, Madurai.

\section{RESULTS}

6900 non-repetitive isolates from 29,275 patients were collected from urine, blood, pus and respiratory samples from January to December 2013-2015. The Gram Positive Cocci and Gram Negative bacterial isolates were 2357 and 4543, respectively. Urine (52\%), blood (7\%), respiratory samples (21\%) and pus (20\%) accounted for the samples from which these samples were obtained. Most common isolates in urine were E. coli (69\%) and Klebsiella species (16\%). Most common isolates in blood were E. coli (38\%), Klebsiella species (25\%) and Salmonella (13\%). Most common isolates in respiratory samples were Klebsiella species (44\%), Pseudomonas (28\%) and E. coli (16\%). Most common isolates in pus samples were E. coli (32\%), Klebsiella species (23\%) and Pseudomonas (27\%). E. coli, Klebsiella species, Pseudomonas from urine, blood, respiratory samples, pus showed high sensitivity to Carbapenems, Piperacillin/Tazobactam, Cefoperazone/Sulbactam and Aminoglycosides. E. coli showed high sensitivity to Nitrofurantoin in urine. E. coli, Klebsiella species, Pseudomonas from urine, blood, respiratory samples and pus showed resistance to 3rd generation Cephalosporins, Fluoroquinolones and Trimeth/Sulfonamides. Overall, ESBL strains increased from $64 \%$ to $66.3 \%$ in 2014, then decreased to $63.7 \%$ in 2015 , while Carbapenem resistant strains increased from $2.4 \%$ to $3.8 \%$.

\section{CONCLUSION}

In this 3 years study we found that there was a progressive increase in antimicrobial resistance in isolates of E. coli, K. pneumoniae and P. aeruginosa, isolated from blood, urine, pus and respiratory culture specimens. ESBL production was seen in the majority of isolates of E. coli and K. pneumoniae. These bacteria showed high sensitivity to Carbapenem, Piperacillin, Tazobactam and Cefoperazone Sulbactam.

\section{KEYWORDS}

Escherichia Coli, Klebsiella Pneumoniae, Pseudomonas Aeruginosa, Antimicrobial Resistance.

HOW TO CITE THIS ARTICLE: Navaneethkrishnan RM, Chandrasegaran S. Evaluation of clinically important gram-negative isolates (escherichia coli, Klebsiella pneumoniae, pseudomonas aeruginosa) and their susceptibility pattern in tertiary care hospital: 3 yr prospective study. J. Evolution Med. Dent. Sci. 2016;5(80):5959-5963, DOI: 10.14260/jemds/2016/1346

\section{INTRODUCTION/BACKGROUND}

In the last three years, the frequency and spectrum of antimicrobial-resistant infections have increased in hospitals. Certain infections that are essentially untreatable have begun to occur as epidemics, both in the developing world and in institutional settings. This resulted in increased morbidity, mortality and health care costs. Enterobacteriaceae are common pathogens responsible for urinary tract infections, abdominal infections and hospital-acquired pneumonia.1,2

Financial or Other, Competing Interest: None.

Submission 29-08-2016, Peer Review 22-09-2016,

Acceptance 28-09-2016, Published 05-10-2016.

Corresponding Author:

Dr. Ram Murgan Navaneethkrishnan,

\#14/2, Siva Sakthi Nagar,

K. Pudur,

Madurai-625007.

E-mail: rammddo@yahoo.co.in

DOI: $10.14260 /$ jemds/2016/1346
ESBLs are enzymes that mediate resistance to extendedspectrum (Third generation) Cephalosporins (e.g. Ceftazidime, Cefotaxime and Ceftriaxone) and Monobactams (e.g. Aztreonam), but do not affect Cephamycins (e.g. Cefoxitin and Cefotetan) or Carbapenems (e.g. Meropenem or Imipenem).

The prevalence of ESBL-producing bacteria have been gradually increasing in tertiary care hospitals according to a 2014 study by Joseph T. Spadafino et al. ${ }^{3}$ ESBLproducing Escherichia coli was $69.4 \%$ in India. Concern has arisen in recent years over increasing rates of resistance to Carbapenems, as there are few therapeutic options for treating infections caused by Carbapenem-resistant bacteria. ${ }^{4}$ Indian single centre studies showed a prevalence of Carbapenem resistance was $12-15 \%$ of Enterobacteriaceae. 5

Hence, the present study was conducted in Apollo Speciality Hospitals, Madurai, South India from January to December 2013-2015. The purpose of the study was to find out the prevalence of ESBL producers and Carbapenem resistance from the clinical isolates of Gram negative bacteria and also their susceptibility to commonly used antibiotics. 
Antibiotics are tested according to CLSI guidelines and our own Antibiotic testing Policy.

\section{MATERIALS AND METHODS}

Type of Study: Cross-sectional, observational, prospective study.

Settings: The study was conducted at Apollo Speciality Hospitals, Lab Services, a tertiary care hospital at Madurai.

Sample Size: 6900 isolates received from various patients' Samples $(29,275)$ with different Pathological condition.

Study Period: From January to December 2013-2015

Inclusion Criteria: Patients without any antibiotic treatment. Exclusion Criteria: Patients already on antibiotic treatments.

\section{METHODOLOGY}

The antimicrobial susceptibility patterns of 6900 nonrepetitive isolates from 29,275 was studied. Samples such as Blood, Urine, Pus, Respiratory samples were collected from patients attending as outpatients and in different clinical departments, respective in patient wards and intensive care units by observing standard specimen collection procedure and aseptic precautions. The Gram Positive Cocci and Gram Negative bacterial isolates were 2357 and 4543 . The clinically important Gram-negative isolates (Escherichia coli, Klebsiella pneumoniae and Pseudomonas aeruginosa) and their susceptibility pattern was studied. Blood Samples were processed with Bactec System. Growth identified in Bactec system was streaked in MacConkey Agar and Blood Agar medium.

The other samples (Urine, pus, respiratory samples) were transported to the lab and streaked on MacConkey Agar and Blood Agar medium. After incubation for $18-24 \mathrm{hrs}$. at $37^{\circ} \mathrm{C}$, the culture plates were examined and bacterial isolates were observed by Gram Staining and motility test. The identification of bacteria was performed by standard protocol by observing MacConkey plates for Lactose/Non- lactose fermenters.

VITEK 2 was used to identify the bacteria and its susceptibility pattern. Antibiotics not included in Vitek 2 were processed by the disc diffusion method (Modified Kirby-Bauer method) using Mueller-Hinton agar.
The results were interpreted as per CLSI (Clinical and Laboratory Standards Institute) guidelines corresponding to that period.6,7

All antibiotic discs were obtained from Oxoid (Oxoid Ltd., Altrincham, Cheshire, United Kingdom) and BD BBL (Becton, Dickinson and Company Ltd., Franklin Lakes, New Jersey, USA).

Extended-Spectrum Beta-Lactamase (ESBL) Screening was calculated with resistance to 3rd generation group of antibiotics (Cefotaxime, Ceftriaxone and Aztreonam) for Enterobacteriaceae.

Carbapenem resistance was calculated with resistance to Carbapenems (Imipenem and Meropenem, Doripenem and Ertapenem) group of antibiotics for Enterobacteriaceae. Interpretation based on VITEK COMPACT 2 as per CLSI guidelines. 6

\section{RESULTS}

\begin{tabular}{|c|c|c|c|c|}
\hline & $\mathbf{2 0 1 3}$ & $\mathbf{2 0 1 4}$ & $\mathbf{2 0 1 5}$ & Total \\
\hline $\begin{array}{c}\text { No. of samples } \\
\text { processed }\end{array}$ & 8955 & 9628 & 10692 & 29275 \\
\hline $\begin{array}{c}\text { No. of culture } \\
\text { positive - Gram } \\
\text { Positive Cocci }\end{array}$ & 1266 & 518 & 573 & $\begin{array}{c}2357 \\
(35 \%)\end{array}$ \\
\hline $\begin{array}{c}\text { No. of culture } \\
\text { positive - Gram } \\
\text { Negative bacilli }\end{array}$ & 1712 & 1535 & 1296 & $\begin{array}{c}4543 \\
(65 \%)\end{array}$ \\
\hline \multicolumn{6}{|c|}{ Total } & & & $\begin{array}{c}\mathbf{6 9 0 0} \\
\mathbf{2 4 \% )}\end{array}$ \\
\hline \multicolumn{3}{|c|}{ Table 1: Total Number of Bacterial Isolates from } \\
Samples (2013-2015) n= 6900
\end{tabular}

Table 1 showed, out of 29,275 samples, 6900 (24\%) isolates were obtained. The Gram Positive Cocci (35\%) and Gram Negative bacterial isolates (65\%) were 2357 and 4543. Conventional methods were used till 2013. Vitek 2 was used from 2014 onwards.

\begin{tabular}{|c|c|c|c|c|c|c|c|c|c|c|c|c|c|c|c|c|c|c|c|c|c|}
\hline \multicolumn{22}{|c|}{ Number of Gram Negative Bacterial Isolates - Sample-Wise $n=4543$} \\
\hline Isolates & \multicolumn{5}{|c|}{ Urine } & \multicolumn{5}{|c|}{ Blood } & \multicolumn{5}{|c|}{ Respiratory } & \multicolumn{5}{|c|}{ Pus } & \multirow[t]{2}{*}{ Total } \\
\hline Year & $\stackrel{m}{N}_{N}^{n}$ & $\stackrel{\mathbb{N}}{\stackrel{H}{N}}$ & $\stackrel{\mathrm{N}}{N}^{n}$ & స్ّ & $\therefore$ & $\stackrel{m}{\stackrel{N}{N}}$ & $\stackrel{+}{\stackrel{N}{N}}$ & $\stackrel{n}{\stackrel{n}{N}}$ & 劳 & $\therefore$ & $\stackrel{m}{\stackrel{N}{N}}$ & $\underset{\sim}{+}$ & ำ & 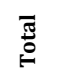 & $\therefore$ & $\stackrel{m}{\tilde{N}}$ & $\underset{\sim}{\stackrel{N}{N}}$ & $\stackrel{n}{\tilde{N}}$ & ఫٓ & s & \\
\hline E. coli & 655 & 522 & 459 & 1636 & 69 & 34 & 34 & 39 & 107 & 38 & 67 & 45 & 43 & 155 & 16 & 111 & 88 & 94 & 293 & 32 & 2191 \\
\hline $\begin{array}{c}\text { Klebsiella } \\
\text { Species }\end{array}$ & 172 & 125 & 95 & 392 & 16 & 16 & 33 & 22 & 71 & 25 & 97 & 244 & 84 & 425 & 44 & 76 & 59 & 73 & 208 & 23 & 1096 \\
\hline \begin{tabular}{|l|} 
Pseudomonas \\
\end{tabular} & 83 & 55 & 59 & 197 & 9 & 16 & 7 & 7 & 30 & 11 & 112 & 101 & 60 & 273 & 28 & 71 & 89 & 88 & 248 & 27 & 748 \\
\hline Proteus Species & 27 & 26 & 7 & 60 & 2.5 & 1 & 0 & 0 & 1 & 0 & 4 & 3 & 2 & 9 & 1 & 17 & 19 & 23 & 59 & 7 & 129 \\
\hline $\begin{array}{l}\text { Citrobacter } \\
\text { Species }\end{array}$ & 39 & 10 & 16 & 65 & 2.5 & 11 & 0 & 0 & 11 & 4 & 33 & 4 & 1 & 38 & 4 & 18 & 5 & 6 & 29 & 4 & 143 \\
\hline Enterobacter & 0 & 7 & 7 & 14 & 0.5 & 0 & 3 & 7 & 10 & 4 & 0 & 5 & 12 & 17 & 2 & 0 & 8 & 17 & 25 & 2 & 66 \\
\hline \begin{tabular}{|l|} 
Acinetobacter \\
\end{tabular} & 3 & 1 & 2 & 6 & 0 & 2 & 2 & 5 & 9 & 3 & 7 & 15 & 27 & 49 & 4 & 4 & 10 & 17 & 31 & 3 & 95 \\
\hline Serratia & 1 & 2 & 6 & 9 & 0.5 & 0 & 2 & 1 & 3 & 1 & 0 & 0 & 3 & 3 & 0 & 0 & 3 & 5 & 8 & 2 & 23 \\
\hline Salmonella & 0 & 0 & 0 & 0 & 0 & 23 & 7 & 7 & 37 & 13 & 0 & 0 & 0 & 0 & 0 & 0 & 0 & 0 & 0 & 0 & 37 \\
\hline Burkholderia & 0 & 0 & 0 & 0 & 0 & 1 & 1 & 1 & 3 & 1 & 0 & 0 & 0 & 0 & 0 & 0 & 0 & 0 & 0 & 0 & 3 \\
\hline \multirow[t]{2}{*}{ Non-fermenter } & 4 & 0 & 0 & 4 & 0 & 0 & 0 & 0 & 0 & 0 & 5 & 0 & 0 & 5 & 1 & 2 & 0 & 0 & 2 & 0 & 12 \\
\hline & 984 & 748 & 651 & 2383 & 100 & 104 & 89 & 89 & 282 & 100 & 325 & 417 & 232 & 974 & 100 & 0 & 281 & 323 & 903 & 100 & 4543 \\
\hline \multicolumn{22}{|c|}{ Table 2: Number of Gram Negative Bacterial Isolates from Samples (2013-2015) $n=4543$} \\
\hline
\end{tabular}


Table 2 showed, the following isolates were isolated from urine, blood, pus and respiratory samples were 52\%, 7\%, 20\%, 21\%. Most common isolates in urine were E. coli (69\%) and Klebsiella species (16\%). Most common isolates in blood were E. coli (38\%), Klebsiella species (25\%) and Salmonella (13\%). Most common isolates in respiratory samples were Klebsiella species (44\%), Pseudomonas (28\%) and E. coli (16\%). Most common isolates in pus samples were E. coli (32\%), Klebsiella species (23\%) and Pseudomonas (27\%).

\begin{tabular}{|c|c|c|c|c|c|c|c|c|c|}
\hline Antibiotics & \multicolumn{3}{|c|}{ E. coli (\%) } & \multicolumn{3}{c|}{ Klebsiella Species (\%) } & \multicolumn{3}{c|}{ Pseudomonas (\%) } \\
\hline & $\mathbf{2 0 1 3}$ & $\mathbf{2 0 1 4}$ & $\mathbf{2 0 1 5}$ & $\mathbf{2 0 1 3}$ & $\mathbf{2 0 1 4}$ & $\mathbf{2 0 1 5}$ & $\mathbf{2 0 1 3}$ & $\mathbf{2 0 1 4}$ & $\mathbf{2 0 1 5}$ \\
\hline Carbapenems & 94.5 & 98.5 & 98.3 & 84 & 90.1 & 88 & 98 & 100 & 86.6 \\
\hline Piperacillin/Tazobactam & 84.5 & 92.7 & 83 & 74 & 72.5 & 57.6 & 94 & 67.9 & 77.7 \\
\hline Cefoperazone/Sulbactam & 83 & 93.4 & 87.5 & 70 & 75 & 64.1 & 77 & 67.3 & 71.1 \\
\hline Aminoglycosides & 86 & 95.4 & 93.1 & 79 & 77.3 & 72.8 & 81 & 88.8 & 66.6 \\
\hline Nitrofurantoins & 73 & 74.1 & 79 & 43 & 67.8 & 39.1 & 0 & 0 & 0 \\
\hline $\begin{array}{c}\text { 3rd Generation } \\
\text { Cephalosporins }\end{array}$ & 34 & 30.6 & 46.4 & 39 & 29.5 & 30.4 & 63 & 65.9 & 55.5 \\
\hline Fluoroquinolones & 33.7 & 34 & 31.6 & 20 & 36.8 & 23.9 & 45 & 47.9 & 53.3 \\
\hline $\begin{array}{c}\text { Trimethoprim/Sulfametho } \\
\text { xazole }\end{array}$ & 44.5 & 45 & 40.3 & 22 & 30.7 & 40.2 & 0 & 0 & 0 \\
\hline Tabis
\end{tabular}

Table 3: Sensitivity Pattern of Antibiotics for Predominant Gram Negative Bacterial Isolates from Urine during $2013-2015$

Table 3 showed, in urine samples, E. coli, Klebsiella and Pseudomonas showed high sensitivity to Carbapenems), Piperacillin/Tazobactam, Cefoperazone/Sulbactam and Aminoglycosides. E. coli showed high sensitivity to Nitrofurantoin. E. coli, Klebsiella and Pseudomonas showed resistance to $3^{\text {rd }}$ generation Cephalosporins, Fluoroquinolones and Trimethoprim/Sulfamethoxazole.

\begin{tabular}{|c|c|c|c|c|c|c|}
\hline \multirow{2}{*}{ Antibiotics } & \multicolumn{3}{|c|}{ E. coli (\%) } & \multicolumn{4}{c|}{ Klebsiella (\%) } \\
\cline { 2 - 8 } & 2013 & 2014 & 2015 & 2013 & 2014 & 2015 \\
\hline Carbapenems & 100 & 100 & 100 & 100 & 89.9 & 95.8 \\
\hline Piperacillin/Tazobactam & 92.6 & 89.5 & 87.5 & 77 & 59.9 & 58.3 \\
\hline Cefoperazone/Sulbactam & 91.1 & 85.5 & 87.5 & 61 & 59.9 & 58.3 \\
\hline Aminoglycosides & 86.9 & 91.6 & 100 & 62 & 81.2 & 75 \\
\hline 3rd Generation Cephalosporins & 29.5 & 28.6 & 47.5 & 41 & 32.6 & 50 \\
\hline Fluoroquinolones & 29 & 26.1 & 37.5 & 50 & 33.1 & 41.6 \\
\hline Trimethoprim/Sulfamethoxazole & 27 & 26.6 & 55 & 39 & 40 & 45.8 \\
\hline Table 4: Sensitivity Pattern of Antibiotics for Predominant Gram Negative Bacterial Isolates from Blood \\
during 2013 to 2015
\end{tabular}

Table 4 showed, in blood samples, E. coli showed high sensitivity to Carbapenems, Piperacillin/Tazobactam, Cefoperazone/Sulbactam and Aminoglycosides. Klebsiella species showed high sensitivity to Carbapenems, Aminoglycosides. E. coli and Klebsiella showed resistance to $3^{\text {rd }}$ generation Cephalosporins, Fluoroquinolones and Trimethoprim/Sulfamethoxazole.

\begin{tabular}{|c|c|c|c|c|c|c|c|c|c|}
\hline Antibiotics & \multicolumn{3}{|c|}{ E. coli (\%) } & \multicolumn{3}{c|}{ Klebsiella (\%) } & \multicolumn{3}{c|}{ Pseudomonas (\%) } \\
\hline & $\mathbf{2 0 1 3}$ & $\mathbf{2 0 1 4}$ & $\mathbf{2 0 1 5}$ & $\mathbf{2 0 1 3}$ & $\mathbf{2 0 1 4}$ & $\mathbf{2 0 1 5}$ & $\mathbf{2 0 1 3}$ & $\mathbf{2 0 1 4}$ & $\mathbf{2 0 1 5}$ \\
\hline Carbapenems & 94.6 & 94.6 & 98.7 & 81.4 & 81.4 & 89.8 & 96.6 & 96.6 & 91.6 \\
\hline Piperacillin/Tazobactam & 87.5 & 87.5 & 77.1 & 66.8 & 66.8 & 62.3 & 79.1 & 79.1 & 88 \\
\hline Cefoperazone/Sulbactam & 82.1 & 82.1 & 78.3 & 67.1 & 67.1 & 62.3 & 78.6 & 78.6 & 85.7 \\
\hline Aminoglycosides & 90.3 & 90.3 & 87.9 & 84.5 & 84.5 & 73.9 & 84.6 & 84.6 & 60 \\
\hline 3rd Generation Cephalosporins & 34.7 & 34.7 & 40.9 & 34.9 & 34.9 & 49.2 & 60.3 & 60.3 & 74.6 \\
\hline Fluoroquinolones & 44.5 & 44.5 & 30.1 & 39.6 & 39.6 & 43.4 & 60.7 & 60.7 & 81.9 \\
\hline Trimethoprim/Sulfamethoxazole & 35.6 & 35.6 & 32.5 & 43.1 & 43.1 & 52.1 & - & - & - \\
\hline
\end{tabular}

\section{Table 5: Sensitivity Pattern of Antibiotics for Predominant Gram Negative Bacterial Isolates from Pus during 2013 to 2015}

Table 5 showed, in pus samples E. coli, Klebsiella and Pseudomonas showed high sensitivity to Carbapenems), Piperacillin/Tazobactam, Cefoperazone/Sulbactam and Aminoglycosides. Pseudomonas showed high sensitivity to Fluoroquinolones and $3^{\text {rd }}$ generation Cephalosporins. E. coli and Klebsiella showed resistance to $3^{\text {rd }}$ generation Cephalosporins, Fluoroquinolones and Trimethoprim/Sulfamethoxazole. 


\begin{tabular}{|c|c|c|c|c|c|c|c|c|c|}
\hline Antibiotics & \multicolumn{3}{|c|}{ E. coli (\%) } & \multicolumn{3}{c|}{ Klebsiella (\%) } & \multicolumn{3}{c|}{ Pseudomonas (\%) } \\
\hline & $\mathbf{2 0 1 3}$ & $\mathbf{2 0 1 4}$ & $\mathbf{2 0 1 5}$ & $\mathbf{2 0 1 3}$ & $\mathbf{2 0 1 4}$ & $\mathbf{2 0 1 5}$ & $\mathbf{2 0 1 3}$ & $\mathbf{2 0 1 4}$ & $\mathbf{2 0 1 5}$ \\
\hline Carbapenems & 100 & 93.7 & 100 & 96 & 92.8 & 100 & 100 & 95.5 & 98.3 \\
\hline Piperacillin/Tazobactam & 100 & 81.8 & 85.7 & 92 & 68.6 & 67.8 & 85 & 83.6 & 89.2 \\
\hline Cefoperazone/Sulbactam & 81 & 89.2 & 88 & 83 & 68.2 & 71.4 & 80 & 76.6 & 92.8 \\
\hline Aminoglycosides & 84 & 83.3 & 95.2 & 83 & 89.3 & 91.6 & 80 & 81.2 & 87.5 \\
\hline 3rd Generation Cephalosporins & 38 & 34.3 & 40 & 57 & 34.9 & 45.2 & 60 & 53 & 71.4 \\
\hline Fluoroquinolones & 13 & 40.9 & 30.9 & 48 & 62.5 & 44 & 60 & 74 & 75 \\
\hline Trimethoprim/Sulfamethoxazole & 25 & 48.8 & 45.2 & 35 & 52.8 & 50 & - & - & - \\
\hline Table 6: Sensitivity Pattern of Antibiotics for Predominant Gram Negative Bacterial Isolates from Respiratory Specimen \\
\hline
\end{tabular}

Table 6 Showed, in respiratory samples E. coli, Klebsiella and Pseudomonas showed high sensitivity to Carbapenems), Piperacillin/Tazobactam, Cefoperazone/Sulbactam and Aminoglycosides. E. coli and Klebsiella showed resistance to 3rd generation Cephalosporins, Fluoroquinolones and Trimethoprim/Sulfamethoxazole.

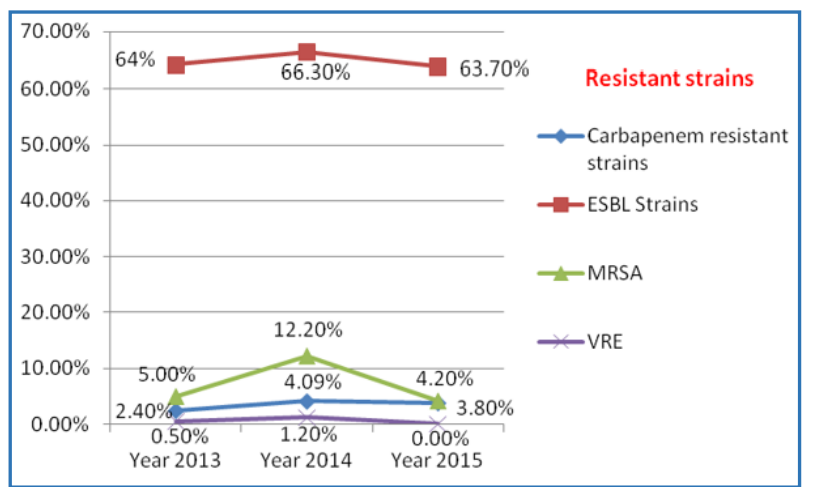

Chart 1: Resistant Strains

Overall, ESBL strains increased from $64 \%$ to $66.3 \%$ in 2014 , then decreased to $63.7 \%$ in 2015 . Overall, carbapenem resistant strains increased from $2.4 \%$ to $3.8 \%$. Resistant strains are identified with Vitek 2 from 2014 onwards. Resistant strains are more prevalent in ICU, CCU and NICU.

\section{DISCUSSION}

This study revealed that Gram negative bacteria (65\%) were more predominant than Gram positive bacteria (35\%). Anitha et al $^{8}$ has reported the incidence of $80.96 \%$ for Gram-negative and $18 \%$ for Gram-positives. Gram negative bacterias were more isolated, which was correlated to present study.

In this study, most common isolates in urine were E. coli (69\%) and Klebsiella species (16\%), Namratha W Nandihal et $\mathrm{al}^{9}$ showed Escherichia coli was the most commonly isolated bacteria and constituted $54.5 \%$, which was followed by Klebsiella species of $21.5 \%$. This study supports the findings of Namratha W Nandihal et al.

In this study, most common isolates in blood were E. coli (38\%), Klebsiella species (25\%) and Salmonella (13\%). Shamim Mumtaz et al $^{10}$ showed in blood samples the most common organism isolated was E. coli followed by Salmonella and Klebsiella. This observation was similar to this study.

In this study, the most common isolates in respiratory samples were Klebsiella species (44\%), Pseudomonas (28\%) and E. coli (16\%). Manikandan et al ${ }^{11}$ showed the prevalence of bacteria spp. isolated were as follows: Klebsiella pneumoniae (38.4\%), Pseudomonas aeruginosa (21\%) and Escherichia coli $(10 \%)$ which correlates with the present study.
In this study E. coli, Klebsiella species, Pseudomonas from urine, blood, respiratory samples, pus showed high sensitivity to Carbapenems, Piperacillin/Tazobactam, Cefoperazone/ Sulbactam and Aminoglycosides.

This is in concordance with the study of Dinesh Kumar et al, ${ }^{12}$ which showed analysis of antimicrobial susceptibility pattern of ESBL producing E. coli isolates from urine and blood demonstrated high susceptibility rates to Imipenem $(100 \%$, $100 \%)$.

Piperacillin/tazobactam (80.48, 70\%), Cefoperazone/sulbactam $(70.73,80 \%)$ and Aminoglycosides such as Amikacin $(73.17,60 \%)$ and Gentamicin $(68.29,60 \%)$, respectively. Al-Zarouni et $\mathrm{al}^{13}$ also demonstrated higher susceptibility rates to Carbapenems and Amikacin. Kutbettin Demirda et $\mathrm{al}^{14}$ showed $100 \%$ sensitive to Imipenem and Meropenem among E. coli and Klebsiella spp. was detected. These observations were highly correlated with us.

In this study, in all samples E. coli isolates showed resistance to $3^{\text {rd }}$ generation Cephalosporins, Fluoroquinolones and Trimeth/Sulfonamides. Marwa E.A. Aly et al 15 showed 81$88 \%$ of MDR E. coli were resistant to Tetracycline, Fluoroquinolones and Sulfonamide/ Trimethoprim. This observation is similar to our study.

In this study, in all samples Klebsiella isolates showed resistance to $3^{\text {rd }}$ generation Cephalosporins, Trimeth/ Sulfonamides and Fluoroquinolones. Philippon A and Babini GS et al $^{16}$ showed that the ESBL Klebsiella was resistant to third generation Cephalosporins as well as the Aztreonam and Fluoroquinolones. This finding correlates with our study.

In this study in all samples Pseudomonas isolates showed resistance to Aminoglycosides, $3^{\text {rd }}$ generation Cephalosporins and Fluoroquinolones. Satyajeet K. Pawar et al ${ }^{17}$ reported that around $70 \%$ of Pseudomonas strains were resistant to Aminoglycosides, Fluoroquinolones and 3rd generation Cephalosporins. This study highly correlates with our study.

In this study overall ESBL strains increased from $64 \%$ to $66.3 \%$ in 2014 , then decreased to $63.7 \%$ in 2015 . Mathai D et al18 noted that the prevalence of ESBLs in India has now reached epidemic proportions, ranging from $62 \%$ to $100 \%$ in E. coli and Klebsiella spp. isolated from skin and soft tissue infections, blood stream infections and respiratory infections as observed in the 10 Indian medical centre SENTRY study. Our study highly correlates with Indian standards. 
High percentage of ESBL producing E. coli has been reported with $60 \%$ in a study done at a tertiary care hospital in Chennai. ${ }^{19}$ These findings correlates with our study.

In this study, overall Carbapenem resistant strains increased from $2.4 \%$ to $3.8 \%$. Camilla Rodrigues ${ }^{20}$ reported that a recent pilot study from a tertiary care centre in central Mumbai that receives referral samples from the city and the State found a steady increase in Carbapenem resistance in Enterobacteriaceae from 0\% in 2006 to 8\% in 2009.

\section{CONCLUSION}

In this 3 years study, we found that there was a progressive increase in antimicrobial resistance in isolates of E. coli, K. pneumoniae and P. aeruginosa isolated from blood, urine, pus and respiratory culture specimens. ESBL production was seen in the majority of isolates of E. coli and K. pneumoniae. Escherichia coli, Klebsiella pneumoniae and Pseudomonas aeruginosa showed high sensitivity to Carbapenem and Piperacillin Tazobactam and Cefoperazone Tazobactam. Hence, for rational and judicial use of existing antibiotics, the Clinicians and Paramedical have to be sensitised with suitable awareness programmes and the present scenario to be brought to the notice of policy makers to formulate the policy and legislation for rational use of Antibiotics.

\section{LIMITATIONS}

This is a single centred study.

\section{ACKNOWLEDGEMENT}

The Authors thank the Administration, Apollo Speciality Hospitals, Lab Services, Madurai, who were kind enough to allow us to conduct this study. The authors gratefully acknowledged the technicians and other members who have helped us to conduct this study successfully.

\section{REFERENCES}

1. Gupta K, Hooton TM, Naber KG, et al. International clinical practice guidelines for the treatment of acute uncomplicated cystitis and pyelonephritis in women: a 2010 update by the infectious diseases society of America and the European society for microbiology and infectious diseases. Clin Infect Dis 2011;52(5):e103-20.

2. Hooton TM, Bradley SF, Cardenas DD, et al. Diagnosis, prevention, and treatment of catheter-associated urinary tract infection in adults: 2009 international clinical practice guidelines from the infectious diseases society of America. Clin Infect Dis 2010;50(5):625-63.

3. Spadafino JT, Cohen B, Liu J, et al. Temporal trends and risk factors for extended-spectrum beta-lactamaseproducing escherichia coli in adults with catheterassociated urinary tract infections. Antimicrob Resist Infect Control 2014;3(1):39.

4. Breilh D, Texier-Maugein J, Allaouchiche B, et al. Carbapenems. J Chemother 2013;25(1):1-17.

5. Porwal R, Gopalakrishnan R, Rajesh NJ, et al. Carbapenem resistant gram-negative bacteremia in an Indian intensive care unit: a review of the clinical profile and treatment outcome of 50 patients. Indian J Crit Care Med 2014;18(11):750-3.
6. Clinical laboratory standard institute. Performance standards for antimicrobial susceptibility testing. Twenty-fifth informational supplement 2015:M100-S25.

7. Kirby WM, Yoshihara GM, Sundsted KS, et al. Clinical usefulness of a single disc method for antibiotic sensitivity testing. Antibiotics Annu 1956-1957:892-7.

8. Anitha M, Pandurangan S, Monisha DM, et al. Prevalence of gram positive and gram negative organisms in various clinical samples. IJAMSCR 2016;4(1):99-107.

9. Nandihal NW. Profile of urinary tract infection and quinolone resistance among escherichia coli and Klebsiella species isolates. Int J Curr Microbiol App Sci 2015;4(7):749-56.

10. Mumtaz S, Zafar S, Ahmad M. Frequency of enteric gram negative rods isolated from various clinical samples. Journal of Islamabad Medical \& Dental College 2012;2:936.

11. Manikandan C, Amsath A. Antibiotic susceptibility of bacterial strains isolated from patients with respiratory tract infections. Int J Pure Appl Zool 2013;1(1):61-9.

12. Kumar D, Singh AK, Ali MR, et al. Antimicrobial susceptibility profile of extended spectrum $\beta$-lactamase (ESBL) producing escherichia coli from various clinical samples. Infect Dis (Auckl) 2014;7:1-8.

13. Al-Zarouni M, Senok A, Rashid F, et al. Prevalence and antimicrobial susceptibility pattern of extendedspectrumbeta-lactamase-producing enterobacteriaceae in the United Arab Emirates. Med Princ Pract 2008;17(1):32-6.

14. Demirda K, Yüce $P$, Özden $M$, et al. Investigation of the susceptibility of extended spectrum beta-lactamase producing escherichia coli and klebsiella spp. Strains to ertapenem and other carbapenems. Mikrobiyol Bul 2011;45(1):28-35.

15. Aly MEA, Essam TM, Amin MA. Antibiotic resistance profile of E. coli strains isolated from clinical specimens and food samples in Egypt. International Journal of Microbiological Research 2012;3(3):176-82.

16. Babini GS, Livermore DM. Antimicrobial resistance amongst klebsiella species collected from intensive care units in southern and western Europe in 1997-1998. J Antimicrob Chemother 2000;45(2):183-9.

17. Pawar SK, Mane PM, Shinde RV, et al. Pseudomonas aeruginosa and its antibiogram from clinical isolates in a tertiary teaching hospital from western Maharashtra, India. Journal of Evidence Based Medicine and Health Care 2014;1(8):574-81.

18. Mathai D, Manoharan A, Vasanthan G. Epidemiology and implications of ESBL. Crit Care Update 2009;14:152-62.

19. Saravanan R, Raveendaran V. Antimicrobial resistance pattern in a tertiary care hospital: an observational study. J Basic Clin Pharm 2013;4(3):56-63.

20. Rodrigues C. Carbapenem-resistant entero-bacteriaceae: a reality check. Regional Health Forum 2011;15(1):83-6. 